\title{
Aproximación ecdótica a los diálogos de Bartolomé Leonardo de Argensola
}

\author{
Germán REDONDO PÉREZ \\ Universidad Complutense de Madrid \\ grperez@pdi.ucm.es
}

\begin{abstract}
RESUMEN
El objetivo principal de este artículo se centra en el análisis de la difusión que los diálogos de Bartolomé Leonardo de Argensola, textos que forman parte del corpus dialógico que edito en mi tesis, han tenido desde que fueron compuestos entre finales del siglo XVI y principios del siglo XVII. Con la información aportada en este trabajo se intenta detallar el conjunto de compilaciones donde fueron agrupados los diálogos, haciendo especial énfasis en las repercusiones que estas circunstancias externas tienen en la elaboración de un texto crítico con el que se pueda ofrecer la versión más fidedigna posible de las composiciones escritas por Argensola.

Además del estudio de los tipos de compilación donde se recogen los cuatro textos, se proponen lo estemas provisionales que han podido configurarse tras el análisis de las variantes contenidas en los testimonios conocidos hasta la fecha.

Por último, se sugieren los testimonios que, teniendo en cuenta el proceso de la recensio, deberían considerarse como textos base para elaborar la edición crítica de cada uno de los diálogos.
\end{abstract}

Palabras clave: ecdótica, Bartolomé Leonardo de Argensola, diálogos, Siglos de Oro.

\begin{abstract}
The main aim of this article is the analysis of the diffusion that the dialogues written by Bartolomé Leonardo de Argensola had. These texts, composed between the last years of the $16^{\text {th }}$ century and the beginning of the $17^{\text {th }}$ century, configure the literary corpus which I study in my doctoral thesis. The information given in this article wants to show an overview of the compilations where these dialogues appear, paying special attention to the repercussions that these external circumstances have in the making of a critical text.

Following a study about the kinds of compilations in which these four texts can be found, provisional stemmae are proposed after an analysis of the known testimonies.

Finally, four base texts are suggested at the end of this article.

Keywords: textual criticism, Bartolomé Leonardo de Argensola, dialogues, Spanish Golden Age.
\end{abstract}

Sumario: 1. Difusión de los textos 2. Propuestas de edición 3. Conclusión. 


\section{Difusión de los textos}

Si se tiene en cuenta la difusión que las obras pertenecientes a géneros prosísticos breves han tenido desde los Siglos de Oro hasta la actualidad, se puede observar que, por lo general, su transmisión ha sido muy dispar.

En el caso del género dialógico resulta habitual encontrar diferentes tipos de difusión. Entre ellos, los más usuales son los siguientes ${ }^{1}$ :

a) Colecciones de diálogos que reúnen varias obras compiladas bajo un criterio unitario basado en la autoría o en la temática común.

b) Diálogos que se han recogido en colectáneas o volúmenes misceláneos que albergan obras de muy diferentes características genéricas, temáticas o autoriales.

c) Diálogos exentos que se han difundido de manera independiente a través de volúmenes que solo contienen un diálogo entendido como obra única, o de facticios en los que se ha encuadernado un texto dialógico junto con otras obras de diferente factura genérica.

Estas tres posibilidades son las que se contemplan en la difusión de los diálogos de Bartolomé Leonardo de Argensola, característica que plantea no pocos problemas a la hora de abordar su edición ${ }^{2}$.

En primer lugar, es importante señalar la carencia de testimonios cercanos a las fechas de redacción, entre finales del s. XVI y principios del s. XVII, que recojan los cuatro diálogos de manera unitaria, a saber, los tres diálogos originales (Menipo litigante, Demócrito y Dédalo) y la traducción lucianesca (Diálogo de Mercurio y la Virtud). Esto supone que, al igual que en el estudio de otros géneros breves, es necesario realizar una minuciosa búsqueda entre una gran variedad de compilaciones para encontrar el corpus íntegro de los testimonios requeridos en el proceso de edición. De este modo, tras el uso de diferentes recursos bibliográficos y la lectura de los estudios existentes sobre la producción dialógica de Argensola, se puede concluir que los textos se hallan dispersos entre siete testimonios manuscritos y tres ediciones impresas ${ }^{3}$.

\footnotetext{
${ }^{1}$ E. Arrigoni; C. Baranda Leturio; M. Fernández Valladares; A. Vian Herrero (2009), pp. 21 y 22 .

${ }^{2}$ La edición crítica de estas obras es una parte de mi tesis doctoral en realización bajo la dirección de Ana Vian Herrero: Imitación y traducción de Luciano en dos escritores áureos: Bartolomé Leonardo de Argensola y Sancho Bravo de Lagunas (ediciones críticas y estudios).

${ }^{3}$ Las fuentes de información bibliográfica donde se precisan más datos acerca de estos testimonios son J. Simón Díaz (1950-1993); R. de Heredia (1891-1894), 1558 y1559 y P. Salvá y Mallén (1992), 728 y 729, aparte de otros recursos electrónicos como el Catálogo Colectivo del Patrimonio Bibliográfico Español (CCPB), donde se puede encontrar información adicional sobre los impresos, o la Biblioteca Virtual del Patrimonio Bibliográfico (BVPB), que facilita un enlace a la reproducción digital del ms. 521 referido en este artículo.
} 
De entre los testimonios manuscritos, adquiere mayor relevancia el ms. 6861 de la Biblioteca Nacional de España. Este testimonio del s. XVII recoge los tres diálogos originales de Bartolomé Leonardo de Argensola. Los textos se encuentran agrupados en forma de colección, por tanto, se puede deducir que existió la intención de que estas obras formaran parte de un corpus aglutinado en un solo volumen. Debido a ello es posible pensar que esta colección de diálogos, aunque escrita en fases diferentes, fuese concebida como una obra unitaria con lazos en común de tipo genérico y temático.

Los diálogos que contiene este testimonio son Menipo litigante, Demócrito y Dédalo, todos ellos copiados de la misma mano en un manuscrito con letra muy cuidada. En favor de la idoneidad de este manuscrito para ser elegido como texto base de tres de los diálogos, además de su antigüedad, las características materiales y el usus scribendi del copista, destaca el reducido número de errores, algo más elevado en el resto de testimonios.

El mismo tipo de compilación se encuentra en el ms. RM-6672 de la Biblioteca de la Real Academia Española. Este manuscrito, procedente de la colección de Rodríguez Moñino, puede datarse en el s. XVIII. De nuevo se vuelven a recopilar en este volumen los tres diálogos del autor aragonés, aunque con diversas variantes respecto al testimonio ms. 6861 conservado en la BNE.

Por su parte, el ms. 4141 de la Biblioteca Nacional de España, con letra del siglo XVII, únicamente copia el diálogo Menipo litigante en un volumen facticio donde también se pueden encontrar algunas composiciones líricas de los hermanos Argensola, una epístola de Bartolomé al Conde de Lemos y poemas de otros autores.

En el ms. Codex 184 de la Universidad de Pennsylvania, testimonio con letra del s. XVII, se leen Menipo litigante y Demócrito, además de la traducción del Diálogo de Mercurio y la Virtud, texto erróneamente atribuido a Luciano durante siglos ${ }^{4}$. Las tres obras se encuentran agrupadas bajo el título Libro de todas las obras que se han podido recoger de los dos hermanos Lupercio y Bartolomé Leonardo, y contiene, además de los diálogos, composiciones poéticas escritas por ambos hermanos.

Las textos dialógicos del ms. Codex 184 de Pennsylvania guardan una estrecha relación con los diálogos recopilados en el ms. 238 de la Universidad de Oviedo, testimonio inédito que no ha sido posible encontrar referenciado en ningún traba-

En cuanto a los estudios consultados, cabe destacar los siguientes trabajos: A. Coroleu (1994); F. Ezpeleta Aguilar (1993 y 1998); O. H. Green (1936 y 1952); T. Grigoriadu (2010); I. Pérez Cuenca (2007 y 2011); L. Schwartz Lerner (1992, 1993, 1998, 2000, 2002, 2006 y el prólogo de su edición conjunta con I. Pérez Cuenca en 2011) y M. O. Zappala (1990).

${ }^{4}$ Véanse los trabajos de F. Ezpeleta Aguilar (1998); A. Coroleu (1994); O. H. Green (1936 y 1952); T. Grigoriadu (2010), p. 35 y M. O. Zappala (1990), pp. 144, 146 y 193. 
jo 5 . En este volumen, que recibe por título general Diálogos de Luciano, se copian el Diálogo de Mercurio y la Virtud, Demócrito y Menipo litigante. Su letra es del s. XVIII y tiene un número considerable de variantes adiáforas en común con el manuscrito de Pennsylvania, sin embargo, el copista de este testimonio comete más errores, de los cuales la mayoría son separativos en relación con el ms. Codex 184. En consecuencia, aunque existe un vínculo entre ambos testimonios, no se puede considerar el manuscrito de Oviedo como un descriptus del volumen de Pennsylvania, pues el elevado número de errores separativos, y la reducida cantidad de errores conjuntivos, hacen que ambos se filien en torno a un subarquetipo.

El ms. 521 de la Biblioteca de Castilla-La Mancha, procedente de la Colección Borbón-Lorenzana, es otro de los testimonios más antiguos. En este códice se localizan los mismos diálogos que en el ms. 6861 de la BNE, aunque compilados junto a obras de diferentes autores y géneros. El conjunto de diálogos parece haber sido copiado por varias manos, siendo de particular interés el primer texto encuadernado en esta sucesión de tres. La copia de esta obra, a saber, Menipo litigante, está compuesta por dos fragmentos de épocas distintas. La mayor parte del manuscrito es del s. XVII, al igual que los folios que recogen el resto de los diálogos, sin embargo, otro copista ha suplido la carencia del principio del diálogo en el manuscrito original con la parte correspondiente que se encuentra publicada en la revista literaria $L a$ Espigadera $^{6}$, adición que aparece delimitada mediante una nota en el vuelto del primer folio donde se puede leer: "Por faltar a este diálogo su principio, según se echa de ver por la antigüedad de la letra, se le copió del que se publicó en el periódico Espigadera, nº 7, año de 1790".

Finalmente, para concluir la presentación de los manuscritos, es necesario referirse al ms. 2844 de la BNE, volumen dedicado a obras de corte lucianesco. En este manuscrito del s. XVIII se recopilan Menipo litigante, Demócrito y Dédalo. Una vez más, los diálogos aparecen encuadernados junto a obras de diferentes autores, en este caso acompañados de una continuación de Las historias verdaderas de Luciano de Samosata, escrita por Francisco de la Reguera, y dos traducciones de Luciano realizadas por Sancho Bravo de Lagunas que reciben por título Discurso de Luciano que no debe darse crédito fácilmente a la murmuración y Almoneda de vidas.

En cuanto a las ediciones que reproducen estas obras, su número se limita a cuatro impresos publicados entre el s. XVIII y el s. XIX.

${ }^{5}$ Detallo las características de este manuscrito desconocido en un artículo en prensa actualmente.

${ }^{6}$ Se puede consultar en el impreso R-7167 de la Biblioteca Nacional de España. Lía Schwartz Lerner e Isabel Pérez Cuenca editan de manera independiente el Menipo litigante de La Espigadera por considerar que no está relacionado con la tradición manuscrita. 
La primera publicación en la que aparece uno de los diálogos vinculados con Bartolomé Leonardo de Argensola es el Ensayo de una Biblioteca de Traductores Españoles. En esta edición de 1778, elaborada por Juan Antonio Pellicer y Saforcada, se edita por primera vez la traducción del Diálogo de Mercurio y la Virtud junto con otras traducciones llevadas a cabo por varios autores ${ }^{7}$. Pellicer toma como modelo de copia el ms. Codex 184 de la Universidad de Pennsylvania y no ofrece cambios significativos con respecto a este manuscrito, por tanto, se puede considerar como un descriptus sin valor en la recensio.

Unos años más tarde, en 1790, se publica el diálogo titulado Menipo litigante, texto original de Argensola que hasta ese momento solo había sido difundido mediante manuscritos. Esta edición vio la luz a través de la ya mencionada revista $L a$ Espigadera, efímera publicación de contenido literario cuyo último número sería impreso en $1791^{8}$. La edición contenida en La Espigadera difiere bastante de los manuscritos que reproducen este diálogo; además, como ya ha quedado dicho más arriba, sirve como modelo de copia para completar la omisión del comienzo del ms. 521 de Toledo, por lo que no se puede prescindir de ella a la hora de hacer una edición crítica del texto.

Cipriano Muñoz y Manzano, Conde de la Viñaza, fue pionero en realizar una edición con cierto rigor filológico de los diálogos de Bartolomé Leonardo de Argensola. En primer lugar, editó Menipo litigante en 1887 en un volumen titulado Algunas obras satíricas inéditas de Lupercio y Bartolomé Leonardo de Argensola9. Partiendo del ms. 4141 de la BNE, reproduce el testimonio manuscrito indicando el modelo de copia que utiliza en su transcripción. Años más tarde, en 1889, publicaría los tres diálogos originales y la traducción lucianesca en el volumen titulado Obras sueltas de Lupercio y Bartolomé Leonardo de Argensola ${ }^{10}$. Además de constituir la primera edición de los cuatro textos dialógicos relacionados con Bartolomé, especifica los testimonios que ha utilizado en la edición y sienta las bases para investigaciones posteriores.

Aparte de estos impresos, cabe destacar la reciente publicación de Lía Schwartz Lerner e Isabel Pérez Cuenca en la que ambas autoras editan los tres diálogos originales de Argensola con el título Sátiras menipeas ${ }^{11}$. En este libro se ofrece al lector una edición que destaca por su profusa anotación y el detalle con el que aparecen referenciados los testimonios que utilizan en la fijación de los textos. Sin embargo,

\footnotetext{
${ }^{7}$ J. A. Pellicer y Saforcada (1778).

${ }^{8}$ Para más información, véase el artículo de Isabel Pérez Cuenca (2011) donde analiza la versión del Menipo litigante publicado en La Espigadera puesta en relación con la historia de esta revista.

${ }^{9}$ B. Leonardo de Argensola (1887).

${ }^{10}$ B. Leonardo de Argensola (1889).

${ }^{11}$ B. Leonardo de Argensola (2011).
} 
el aparato de variantes no cuenta con las lecturas contenidas en el ms. 238 de la Universidad de Oviedo debido a su desconocimiento. Además, no se emplea una metodología de edición crítica definida, evidenciándose particularmente esta carencia en las fases de stematis codicum y constitutio textus.

\section{Propuestas de edición}

La gran variedad de tipos de difusión que han recibido los cuatro diálogos implica plantearse diferentes cuestiones a la hora de iniciar el proceso de edición crítica. En primer lugar es necesario decidir si los cuatro textos merecen una edición conjunta o si, por el contrario, cada una de las obras debe ser editada de forma independiente. Como se ha precisado en el apartado anterior, de manera excepcional se encuentra una compilación que recoja los tres diálogos originales junto con la traducción lucianesca, sin embargo, es habitual que se recopilen tres diálogos en un solo volumen o, como en el caso del ms. 521 de Toledo, el ms. Codex 184 de Pennsylvania o el ms. 238 de Oviedo, en compilaciones que carecen de uno de los escritos originales pero que incorporan la traducción.

Hay que tener en cuenta que cada uno de los textos fue escrito en años diferentes e incluso, como se puede concluir tras la lectura del diálogo Dédalo, con propósitos que se alejan del mero fin literario ${ }^{12}$. A esto hay que sumarle el hecho de que el Diálogo de Mercurio y la Virtud sea una traducción, y no un texto de producción propia creado por Argensola. En consecuencia, resulta razonable cuestionarse si es apropiado editar los textos de manera conjunta, pues, como se puede ver, aparentemente son más numerosas las diferencias que las similitudes entre los cuatro textos.

Sin embargo, hay que considerar los elementos en común que mantienen entre sí, más relevantes que las diferencias que los separan. En primer lugar, los cuatro textos pertenecen al género dialógico $\mathrm{y}$, por tanto, tratándose de las únicas obras escritas en forma de diálogo por el autor aragonés, parece lógico que todas ellas aparezcan antologizadas y estudiadas en un solo volumen para ofrecer una perspectiva global de las incursiones del autor en este género. Además, si bien es cierto que cada texto trata materias diferentes, desarrolladas mediante tramas y personajes independientes, no deja de ser posible establecer un nexo en común que se fundamente en las características lucianescas de todos ellos. Este rasgo resulta determinante en la confección de volúmenes como el ms. 2844 de la BNE, donde el compilador entiende que tres de los diálogos deben ir agrupados en un volumen dedicado a obras de tipo lucianesco.

Con respecto a la traducción del texto del autor samosatense, es importante destacar su compleja difusión desde que fue creado hasta que Bartolomé Leonardo de Argensola lo trasladó al castellano. Desde este punto de vista, la edición del Diálogo de Mercurio y la Virtud junto con los textos originales del autor aragonés no solo com-

${ }^{12}$ F. Ezpeleta Aguilar (1998); O. H. Green (1952) y L. Schwartz Lerner (1998). 
pletaría la panorámica sobre la atención que este escritor dedicó al género y, en particular, al diálogo de características lucianescas, sino que ayudaría a esclarecer, de una manera más analítica de la permitida por los límites de este artículo, la difusión de un texto que solo en España recibió varias traducciones y que en la mayoría de ellas, como en el caso que nos ocupa, fue erróneamente atribuido a Luciano.

Otro problema que se deriva del tipo de difusión que han tenido estas obras se manifiesta a la hora de realizar un estema con el que se puedan filiar los testimonios para abordar su edición crítica. La causa de esta dificultad radica en que no existe un testimonio que recoja los cuatro diálogos hasta la edición impresa realizada por el Conde de la Viñaza en 1889, sino que únicamente fueron transmitidos a través de volúmenes en los que se copian los tres diálogos originales, como en el caso de los mss. 6861, RM-6672 ó 2844, volúmenes facticios donde solo aparece inserto un diálogo, como en el ms. 4141, o mediante manuscritos donde se encuentran dos de los diálogos originales, no siendo siempre los mismos títulos, acompañados de la traducción lucianesca, como se puede observar en los mss. Codex. 184, 238 y 521.

Esto implica que no se pueda realizar un estema partiendo de un solo testimonio que agrupe los cuatro diálogos, puesto que no lo hay, sino que es necesario individualizar cada uno de los textos y colacionar de manera independiente los testimonios que han difundido la obra sin prestar atención, desde el punto de vista metodológico, al resto de diálogos junto a los que han sido compilados. En consecuencia, la edición crítica de estas obras merece estemas independientes que muestre las filiaciones entre testimonios.

Partiendo de este planteamiento metodológico, es necesario incluir en la recensio los siguientes testimonios para cada diálogo:

1. Menipo litigante: ms. 6861, ms. RM-6672, ms. 4141, ms. Codex. 184, ms. 238, ms. 521 y ms. 2844. Además de estos testimonios manuscritos, es necesario contar en el proceso de edición con el testimonio de Menipo litigante que se recoge en la revista La Espigadera, pues, a pesar de ser un impreso tardío, aporta una versión muy diferente del resto de testimonios y, por tanto, se podría conjeturar que o bien procede de un modelo de copia desconocido por el resto de copistas que han transmitido el diálogo o bien ha sido manipulado intencionadamente.

2. Demócrito: ms. 6861, ms. RM-6672, ms. Codex. 184, ms. 238, ms. 521 y ms. 2844.

3. Dédalo: ms. 6861, ms. RM-6672 y ms. 2844.

4. Diálogo de Mercurio y la Virtud: ms. Codex. 184, ms. 238 y ms. 521. Queda descartada la edición publicada por Pellicer por tratarse de un descriptus del ms. Codex. 184.

Teniendo en cuenta las variantes que resultan de la colación de todos los testimonios, es posible ofrecer una primera conclusión general sobre el conjunto de estemas del corpus dialógico. La principal característica de los cuatro estemas es que en ninguno de ellos se puede establecer una filiación de los testimonios que han transmitido la obra en torno a un arquetipo, pues, aunque tienen variantes adiáforas en común, no albergan ningún error conjuntivo que permita realizar dicha agrupa- 
ción. Por tanto, si bien se podría pensar en la existencia de ascendentes comunes como modelo intermedio entre el original y los testimonios conservados, más aún si se analizan las variantes adiáforas, metodológicamente resulta inviable situar en el estema un arquetipo común para todos ellos si no se tiene constancia de error conjuntivo alguno ${ }^{13}$.

En consecuencia, cada uno de los testimonios conservados, con excepción del ms. Codex 184 y el ms. 238, que se encuentran agrupados por un subarquetipo común, deben ser presentados en el estema como procedentes directamente del original, aunque con las explicaciones oportunas en el prólogo de la edición.

Tras analizar las variantes de cada testimonio y establecer la filiación a través de errores, se puede llegar a las siguientes propuestas estemáticas:

\section{Estema de Menipo litigante}

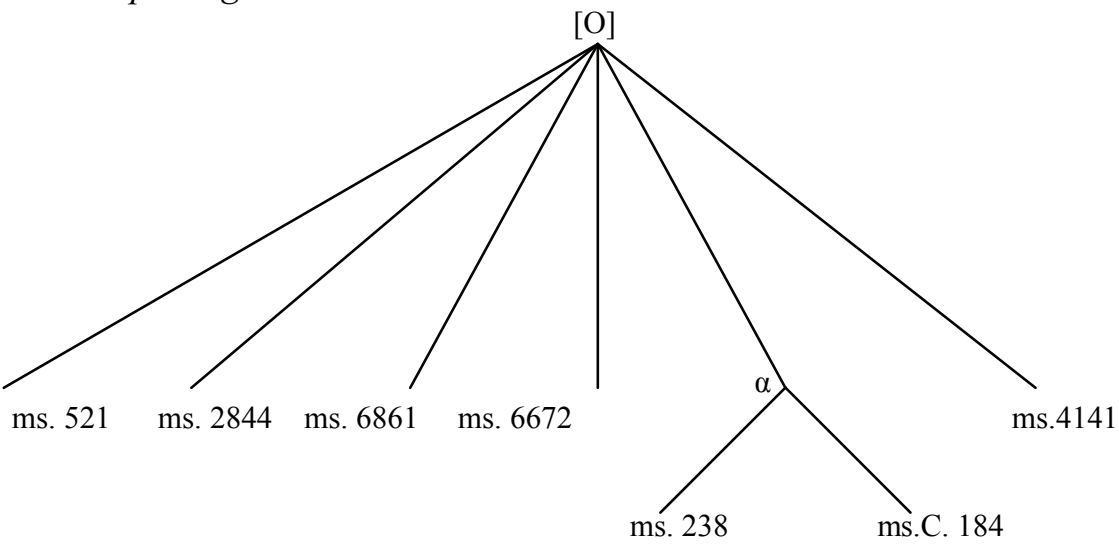

${ }^{13}$ M. A. Pérez Priego (1997), p. 63. 


\section{Estema de Demócrito}

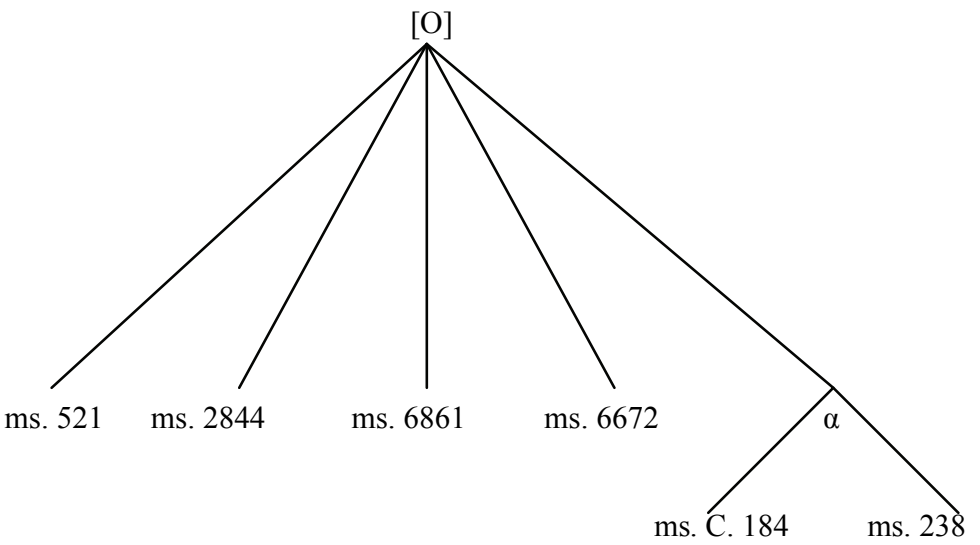

\section{Estema de Dédalo}

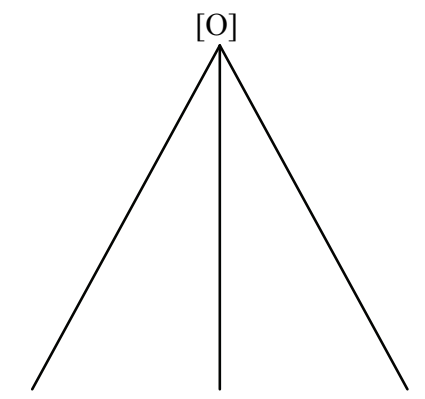

ms. 2844 ms. 6861 ms. 6672 


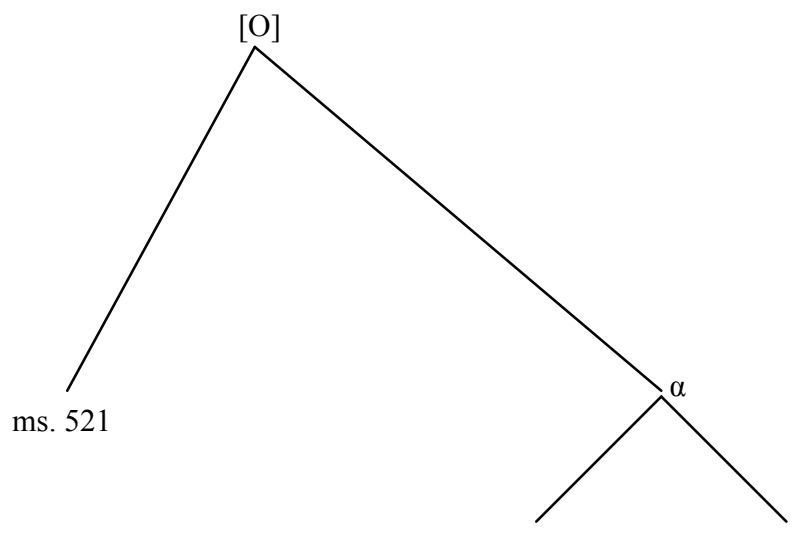

ms. C. 184 ms. 238

Según los datos extraídos del proceso de la recensio, se sugiere el ms. 6861 como texto base para la edición de los diálogos Menipo litigante, Demócrito y Dédalo. Este testimonio del s. XVII no es solo uno de los más antiguos y más próximos al usus scribendi del autor, sino que además posee un ínfimo número de errores en comparación con el resto de testimonios, siendo poco significativos en todos los casos.

En cuanto a la traducción, el ms. Codex 184, debido a su menor número de errores en relación con los otros testimonios que copian el diálogo, a saber, el ms. 521 y el ms. 238, se revela como el más recomendable para su uso como texto base en la edición de esta obra. Esta hipótesis se ve apoyada, como en el caso anterior, por el análisis gramatical del testimonio y la antigüedad del manuscrito.

\section{Conclusión}

Según el estudio de la difusión que han tenido los diálogos que ocupan el contenido del presente trabajo, parece necesaria una edición de mayor complejidad en la que se ahonde en las diversas variantes de cada testimonio y, en resumen, donde se ofrezca un análisis exacto sobre la transmisión que han tenido estas obras con el objetivo de reconstruir un texto lo más fiel posible a lo que pudo ser el original.

En este sentido, se puede considerar que una edición crítica textual siguiendo las premisas de la metodología lachmanniana es el mejor recurso filológico que un editor puede emplear para ofrecer al lector contemporáneo una edición rigurosa en la que todas las particularidades de estos diálogos queden estudiadas. 
Además de los aspectos crítico-textuales, el estudio de estas obras supone la recuperación de unos textos no tan conocidos que fueron creados por uno de los autores más importantes en lengua española. Asimismo, si se tienen en cuenta las características lucianescas de este corpus, se pueden establecer los primeros puntos de partida para abordar la recepción e influencias que el autor samosatense tuvo en la España literaria de entre finales del siglo XVI y mediados del siglo XVII, tema menos estudiado que la trascendencia de Luciano en la edad dorada del género dialógico.

\section{Obras citadas}

ARrigoni, Eleonora; BARANDA LeTURIO, Consolación; FernÁndeZ VALlaDARES, Mercedes; VIAN HERRERO, Ana: Dialogyca BDDH (Biblioteca Digital de Diálogo Hispánico). Manual de procedimiento de trabajo normalizado, Madrid, IUMP (UCM), Febrero de 2009 ( $3^{\mathrm{a}}$ versión).

COROLEU, Alejandro: "El Momo de Leon Battista Alberti: una contribución al estado de la fortuna de Luciano en España", Cuadernos de Filología Clásica. Estudios latinos, 7, (1994), pp. 173-183.

EzPeletA Aguilar, Fermín: "Los diálogos lucianescos de Bartolomé Leonardo de Argensola y la tradición del género dialogal clásico", en Humanismo y pervivencia del mundo clásico: actas del I Simposio sobre Humanismo y pervivencia del mundo clásico, al cuidado de José María Maestre Maestre y Joaquín Pascual Barea, Cádiz, Instituto de Estudios Turolenses, Universidad de Cádiz, 1993, pp. 441-450.

: "La traducción del diálogo de Alberti Virtus por Bartolomé Leonardo de Argensola", en Humanismo y pervivencia del mundo clásico: Homenaje al profesor Luis Gil, al cuidado de José María Maestre Maestre y Joaquín Pascual Barea, Cádiz, Universidad de Cádiz, 1997, pp. 995-1004.

: "El diálogo Dédalo, de Bartolomé Leonardo de Argensola", en Actas del

VII congreso internacional de la Asociación Española de Semiótica, al cuidado de Túa Blesa, Zaragoza, Universidad de Zaragoza, 1998, pp. 306-313.

GreEn, Otis H.: "Notes on the Lucianesque Dialogues of Bartolomé Leonardo de Argensola", Hispanic Review, III (1936), pp. 275-295.

: "Bartolomé Leonardo de Argensola y el Reino de Aragón", Archivos de Filología Aragonesa, 4 (1952), pp. 7-112.

GRIGORIADU, Teodora: La obra de Luciano Samosatense, orador y filósofo excelente. Manuscrito de la Biblioteca Menéndez y Pelayo: edición y estudio. Madrid, Universidad Complutense de Madrid (Tesis doctorales en e-prints), 2010. Próximo a ser publicado en papel por la Real Sociedad Menéndez Pelayo de Santander. 
HerediA, Ricardo: Catalogue de la Bibliothèque de M. Ricardo Heredia, Comte de Benahavis, París, Em. Paul, L. Huard et Guillemin, 1891-1894. 4v, 1558 y 1559. LEONARDO DE ARGENSOLA, Bartolomé: La Espigadera, 7, Madrid, Imprenta de Blas de Román, 1790.

: Algunas obras satíricas inéditas de Lupercio y Bartolomé Leonardo de Argensola, ed. del Conde de la Viñaza, Zaragoza, Imprenta del Hospicio Provincial, 1887.

: Obras sueltas, ed. del Conde de la Viñaza, Madrid, Imprenta y Fundición de M. Tello, 1889.

: Sátiras menipeas, ed. de Lía Schwartz Lerner e Isabel Pérez Cuenca, Zaragoza, Prensas Aragonesas, 2011.

PELliCER Y SAFORCADA, José Antonio: Ensayo de una Biblioteca de Traductores Españoles, Madrid, Antonio Sancha, 1778.

PÉREZ CUENCA, Isabel: "La obra manuscrita de los hermanos Argensola", en Actas del XV congreso de la Asociación Internacional de Hispanistas: 'Las dos orillas', al cuidado de Beatriz Mariscal y María Teresa Miaja de la Peña, México, Fondo de Cultura Económica, 2007, II, pp. 401-410.

: "Menipo litigante, diálogo satírico de Bartolomé Leonardo de Argensola, en la prensa reformista", Acta Poetica, 32-2 (2011), pp. 177-210.

PÉREZ PRIEGO, Miguel Ángel: La edición de textos, Madrid, Síntesis, 1997.

SAlvá y MAllén, Pedro: Catálogo de la Biblioteca de Salvá... enriquecido con la descripción de otras muchas obras, de sus ediciones, etc., Valencia, Imp. de Ferrer de Orga, 1872. 2v. (Ed. Facsímil: Madrid, Julio Ollero, 1992), 728 y 729.

SCHWARTZ LERNER, Lía: "El diálogo en la cultura áurea: de los textos al género", Ínsula: revista de letras y ciencias humanas, 542 (1992), pp. 1-28.

: "Modelos clásicos y modelos del mundo en la sátira áurea: Los diálogos de Bartolomé Leonardo de Argensola", en Estado actual de los estudios sobre el Siglo de Oro: Actas del II congreso internacional de hispanistas del Siglo de Oro, al cuidado de Manuel García Martín, Salamanca, Universidad de Salamanca, 1993, I, pp. 75-94.

: "Las alteraciones aragonesas y los Argensola", en Felipe II (1598-1998). Europa y la Monarquía Católica, al cuidado de José Martínez Millán, Madrid, Parteluz, 1998, II, pp. 815-832.

: "La representación del poder en la sátira áurea: del rey y sus ministros en el Dédalo de Argensola y en los Sueños de Quevedo", Le pouvoir au miroir de la littérature en Espagne aux XVIe et XVIIe siècles, al cuidado de Agustín Redondo, París, Université de la Sorbonne, 2000, pp. 33-48.

: "Bartolomé Leonardo de Argensola: las voces satíricas de un humanista aragonés", Caliope, VIII, 2 (2002), pp. 51-74.

: "La fábula mitológica y la sátira: Menipo litigante de Bartolomé Leonardo de Argensola", en Homenaje a Ana María Barrenechea, al cuidado de Melchora Romanos et alii, Buenos Aires, Eudeba, 2006, pp. 431-442. 
Germán Redondo Pérez Aproximación ecdótica a los diálogos de Bartolomé Leonardo de Argensola

SimÓN DÍAZ, José: Bibliografia de la Literatura Hispánica, Madrid. CSIC, 19501993, 16v.

ZAPPALA, Michael. O.: Lucian of Samosata in the Two Hesperias: an essay in literary and cultural translation. Potomac-Maryland, Scripta Humanistica, 1990. 